\title{
Combined Interhemispheric and Transsylvian Approach for Resection of Craniopharyngioma
}

\section{Tomohiro Inoue ${ }^{1}$ Hideaki Ono ${ }^{2}$ Akira Tamura ${ }^{2}$ Isamu Saito ${ }^{2}$}

\footnotetext{
${ }^{1}$ Department of Neurosurgery, NTT Medical Center Tokyo, Tokyo, Japan

2 Department of Neurosurgery, Fuji Brain Institute and Hospital, Fujinomiya Shi, Japan
}

\author{
Address for correspondence Tomohiro Inoue, MD, PhD, Department \\ of Neurosurgery, NTT Medical Center Tokyo, 5-9-22 Higashi Gotanda \\ Shinakawa-ku, Tokyo 141-0022, Japan \\ (e-mail: t.inouenttnsu@gmail.com).
}

J Neurol Surg B 2018;79(suppl S3):S251.

\section{Abstract Keywords \\ - interhemispheric approach \\ - lamina terminalis \\ - pterional approach \\ - craniopharyngioma}

We present a 37-year-old male case of cystic suprasellar huge craniopharyngioma, who presented with significant memory disturbance due to obstructive hydrocephalus. Combined interhemispheric and pterional approach was chosen to resect huge suprasellar tumor. Interhemispheric trans-lamina terminalis approach was quite effective to resect third ventricular tumor, while pterional approach was useful to dissect tumor out of basilar perforators and stalk.

The link to the video can be found at: https://youtu.be/BoYIPa96kdo.
Conflict of Interest

None.

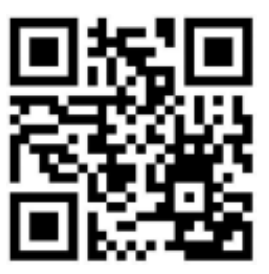

received

September 18, 2017 accepted

December 18, 2017

published online

February 20, 2018

www.thieme.com/skullbasevideos

www.thieme.com/jnlsbvideos

DOI https://doi.org/

10.1055/s-0038-1625968. ISSN 2193-6331.
๑) 2018 Georg Thieme Verlag KG
Stuttgart · New York

License terms

(ㄷ) (i) $\ominus$ (8) 